\title{
Aplicación de la hipótesis de paridad de poder adquisitivo en el pronóstico de la tasa de cambio del peso colombiano contra el dólar estadounidense
}

\author{
JIMÉNEZ-MÉNDEZ, EDGAR RICARDO \\ Universidad Jorge Tadeo Lozano (Bogotá, Colombia) \\ Correo electrónico: edgarr.jimenezm@utadeo.edu.co \\ Aguilera PEÑA, NicolÁs \\ Universidad Jorge Tadeo Lozano (Bogotá, Colombia) \\ Correo electrónico: nicolas.aguilerap@utadeo.edu.co
}

\begin{abstract}
RESUMEN
Este paper muestra el pronóstico de la tasa de cambio entre el peso colombiano y el dólar estadounidense (en adelante dólar) desde Diciembre del 2019 hasta Diciembre del 2020, basado en un modelo VEC (Vector Error Correction) a partir de la hipótesis de paridad de poder adquisitivo débil. Con esto, se propone que la tasa de cambio se ajusta no solamente para equilibrar las diferencias en precios entre Estados Unidos y Colombia, sino también para equilibrar una tercera variable, en este caso el precio del petróleo WTI. Dado que la principal fuente de ingresos por exportaciones para Colombia son los recursos petroleros, se consideró valioso usar las cotizaciones del WTI como proxy para estimar la probable dirección que siga la tasa de cambio en el corto y medio plazo.
\end{abstract}

Palabras clave: pronóstico, peso colombiano, petróleo WTI, Modelos VEC, paridad. Clasificación JEL: G17.

MSC2010: 6202; 65C60; 91G70; 91G80. 


\title{
Application of the purchasing power parity hypothesis in the forecast of the exchange rate of the Colombian peso against the US dollar
}

\begin{abstract}
This paper shows the forecast of the exchange rate between the Colombian peso and the US dollar (hereinafter the dollar) from December 2019 to December 2020, based on a VEC (Vector error correction) model based on the parity hypothesis of weak purchasing power. With this, it's proposed that the exchange rate is adjusted not only to balance the differences in rates between the United States and Colombia, but also to balance a third variable, in this case WTI crude oil price. Caused by that the main source of export earnings for Colombia has its source in oil resources, it was considered valuable to use WTI quotes as a proxy to estimate the likely direction of the exchange rate in the short and medium term.
\end{abstract}

Keywords: forecast, Colombian peso, WTI Oil, VEC Models, parity.

JEL classification: G17.

MSC2010: 6202; 65C60; 91G70; 91G80.

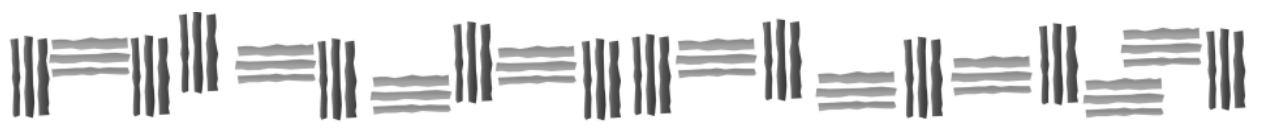




\section{Introducción y revisión bibliográfica.}

La hipótesis de paridad de poder adquisitivo (PPP por sus siglas en inglés) es acogida por la mayoría de economistas como una explicación racional de las tasas de cambio en respuesta a la diferencia de precios en dos países distintos (Mankiw, 2016). Su lógica está centrada en que los agentes racionales explotan las oportunidades de arbitraje internacional en precios a través del comercio. De esta forma los efectos de la entrada y salida de divisas producto del intercambio equilibran los precios internacionales a través de la tasa de cambio (Lipschitz \& Schadler, 2019).

Sin embargo, esta hipótesis ha sido ampliamente refutada como lo explica Wang (2009), en su amplia revisión literaria. En esta muestra que Krugman (1978) no encuentra evidencia de que la hipótesis de PPP se mantenga para el periodo entre guerras (primera y segunda guerra mundial) y de flotación reciente (régimen flexible después de la salida de Estados Unidos del pacto de Bretton Woods en 1971). Frenkel (1981) no encuentra evidencia suficiente para sostener que la hipótesis de PPP relativo se mantiene y argumenta que puede ser consecuencia de rigideces comerciales, tales como políticas arancelarias. Por otra parte, Serletis y Gogas (2004) encuentran que la evidencia a favor del PPP es poco robusta.

En otras cosas siguiendo el hilo de Wang (2009) a favor del PPP se puede encontrar que Cumby y Obstfeld (1984) aportan evidencia contundente para la tasa de cambio entre Estados Unidos y Canadá para el periodo de flotación reciente. Por su parte, Holmes y Wang (2005) encuentran que para una muestra de países africanos se cumple la hipótesis cuando se analiza la convergencia de largo plazo. Sucesivamente Papell y Prodan (2006) encuentran que modelando las divisas de 14 países y teniendo en cuenta los cambios estructurales, no se rechaza la hipótesis de paridad por poder adquisitivo.

En cuanto a la hipótesis de cointegración entre la razón de precios y la tasa de cambio puede encontrarse que, sin salir de la revisión hecha por Wang (2009), Taylor (1988), Patel (1990) y Kim (1990) usan el método de cointegración de dos etapas de Engle y Granger (1987) donde encuentran resultados poco satisfactorios en vista de que no se satisface el equilibrio de largo plazo; sin embargo persistiendo en la hipótesis de cointegración mediante el método de Johansen y Juselius (1990), McDonald (1993), Cochrane y DeFina (1995) y Jacobson y Nessen (2004) aportan evidencia de que los precios relativos y la tasa de cambio demuestran convergencia conjunta en el largo plazo.

Respecto a los usos del modelo VEC, Hernández, Mesa y Vélez (2009) logran encontrar pronósticos consistentes en modelos VAR usando la hipótesis de PPP. Cerquera, Gómez y Arias (2018) demuestran la inexistencia de co-movimiento entre la tasa de cambio del peso colombiano y el dólar estadounidense con los precios relativos. Kugler y Lenz (1993) muestran cómo se cumple la hipótesis de PPP como una propiedad de largo plazo para la libra, la lira, la corona noruega, el schilling, el escudo y la peseta, mientras que parece no mantenerse para la corona sueca, el franco belga, y el dólar con el dólar canadiense.

\section{Procedimiento.}

Teniendo en cuenta la revisión literaria presentada anteriormente se considera que un buen modelo para el pronóstico de la tasa de cambio, teniendo en cuenta la paridad de poder adquisitivo como elemento fundamental, puede ser un modelo VEC que contemple la hipótesis débil PPP al incluir el precio del WTI como una variable adicional que logra equilibrar la relación conjunta con los precios relativos y la tasa de cambio pesos colombianos por dólar. Esta idea surge de lo planteado por McDonald (1993) donde la estacionariedad de las desviaciones de una divisa respecto a una cesta de otras es tenida en cuenta al momento de validar la paridad por poder adquisitivo.

De esta manera se plantea que cuando la tasa de cambio no está corrigiendo para aliviar las diferencias entre los precios nacionales y extranjeros, debe ser porque los efectos del WTI sobre la 
balanza de pagos Colombiana mantienen la tasa de cambio real en "desequilibrio". Lo último implicaría que aún en presencia de desajustes en PPP, la relación entre los precios el WTI y la tasa de cambio debería ser estacionaria.

La razón para asumir que el WTI es una variable que puede estar explicando estos choques en la tasa de cambio real viene de la relevancia que la venta de crudo ha tenido dentro de las exportaciones colombianas (Gráfico 1). Por ejemplo, dentro de los últimos 13 años el peso mínimo del sector petróleo, productos derivados del petróleo y productos convexos dentro de las exportaciones colombianas ha sido de $25,76 \%$, mientras que el promedio en este lapso temporal ha sido de $39,43 \%$. Esto revela que, en promedio, con todo lo demás constante, cada $1 \%$ de cambio en el precio de estas exportaciones ha tenido que contribuir a un cambio inmediato del $0,39 \%$ en el valor de las exportaciones, o lo que es lo mismo en la entrada de dólares por cuenta de exportaciones.

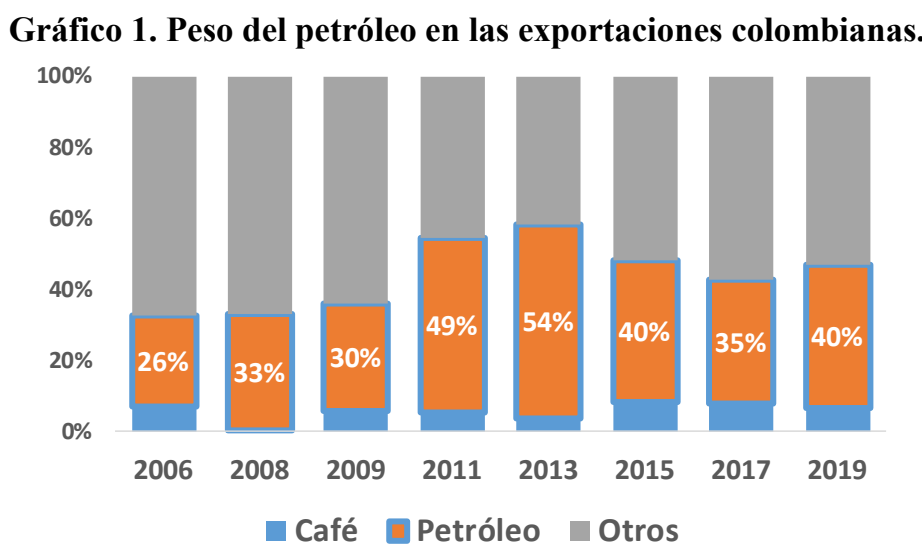

Fuente: Elaboración propia a partir de datos del DANE (DANE, 2019).

Otro aspecto relevante del precio del petróleo, es la manera en que puede impactar la inflación, ya que los combustibles derivados del petróleo impactan el sector energético de la cesta familiar (Gregorio, 2012). Por lo tanto, es racional asumir que, además de afectar la balanza de pagos, también puede afectar los precios que perciben los agentes económicos, tanto en Colombia como en Estados Unidos.

Por otra parte, al observar los efectos de la tasa de cambio sobre el WTI y la inflación se puede encontrar que si un choque exógeno diferente al del precio del petróleo, afectase al dólar a nivel mundial, los productores de petróleo, en busca de maximizar sus ganancias en moneda local, podrían responder variando la cantidad producida (Amuzegar, 1978). Ahora bien, ya que adaptar la producción toma tiempo en consecuencia a que cambiar los factores de producción puede demorarse, entonces puede deducirse que si el choque exógeno al dólar se ve reflejado en la tasa de cambio colombiana, el precio del WTI podría responder de manera significativa a retardos temporales en la tasa de cambio.

El mecanismo de ajuste se daría, por ejemplo, en un aumento de la producción petrolera mundial a largo plazo si el nivel del dólar a nivel mundial sube y les permite a los petroleros recibir más dólares por cada barril; eventualmente este aumento en la producción haría que el precio del WTI corrija a la baja. Por el contrario, si bajase el dólar la reducción de costos, producto de la conducta maximizadora de la firma (Varian, 1992), incentivaría a las empresas a reducir la producción, lo que eventualmente por la ley de oferta y demanda llevaría a que el precio del petróleo corrigiera al alza.

Un ejemplo de la similitud entre el índice dólar calculado por la FED (Federal Reserve Bank of St Louis, 2019), que mide el precio promedio del dólar ponderado por el valor de los bienes y servicios intercambiados para los mayores socios comerciales de Estados Unidos y la tasa de cambio en 
Colombia, además de una elevada correlación con el precio del petróleo, como puede observarse en el Gráfico 2. De forma análoga en el Gráfico 3 se muestra la relación entre el índice dólar (inverso) y el precio del petróleo.

\section{Gráfico 2. Índice del Dólar (FRED) contra tasa de cambio USD/COP.}

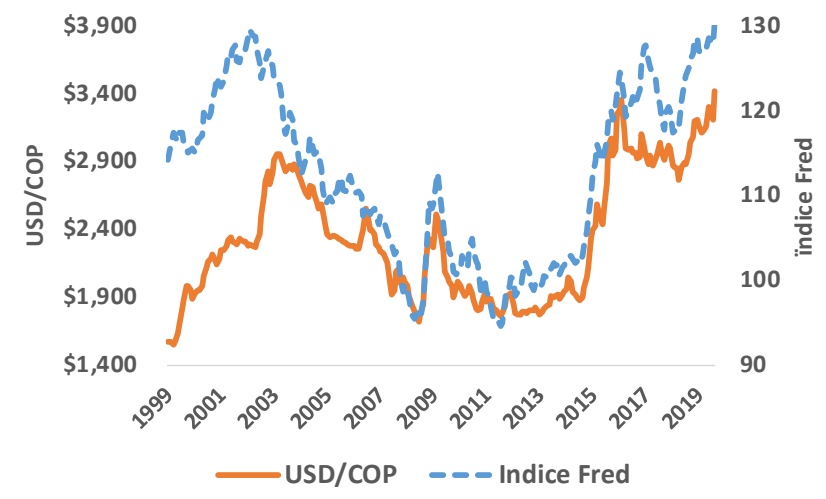

Fuente: Elaboración propia con datos de la FRED de ST Louis y Bloomberg.

Gráfico 3. Índice del Dólar (FRED) - Inverso contra petróleo WTI.

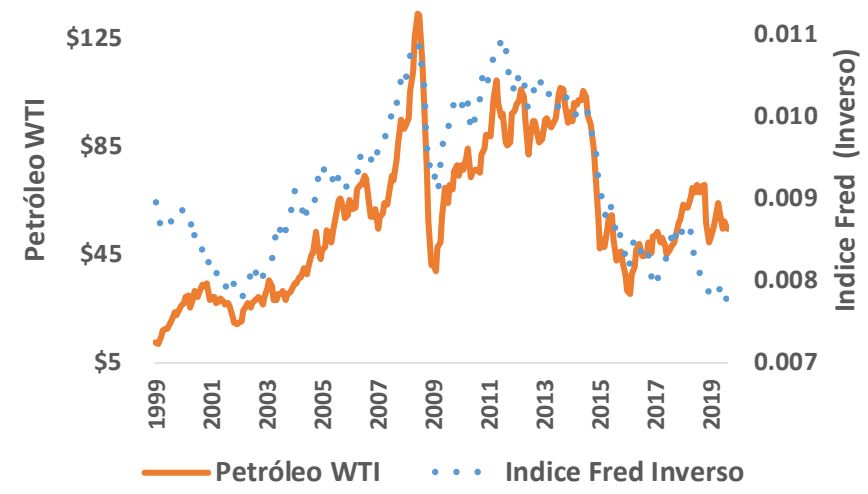

Fuente: Elaboración propia con datos de la FRED de ST Louis y Bloomberg.

En cuanto a los efectos de la Razón de precios sobre el WTI se deduce la existencia de una relación, debido a que la inflación en Estados Unidos está ligada a la productividad económica (Garín, Lester, \& Sims, 2018), entonces en épocas de alto crecimiento la demanda por bienes y servicios, entre ellos el petróleo, deberían generar presiones inflacionarias además de la valorización en los precios del petróleo. De esta manera, la correlación de la inflación estadounidense con el WTI debería transmitirse a la razón de precios. Esta relación se puede ver en el Gráfico 4, donde se da un comportamiento similar en cuanto a la dirección de las cotizaciones del combustible fósil y las variaciones en los índices generales de precios al consumidor estadounidense. 


\section{Gráfico 4. Variaciones cotización WTI contra inflación en los Estados Unidos.}

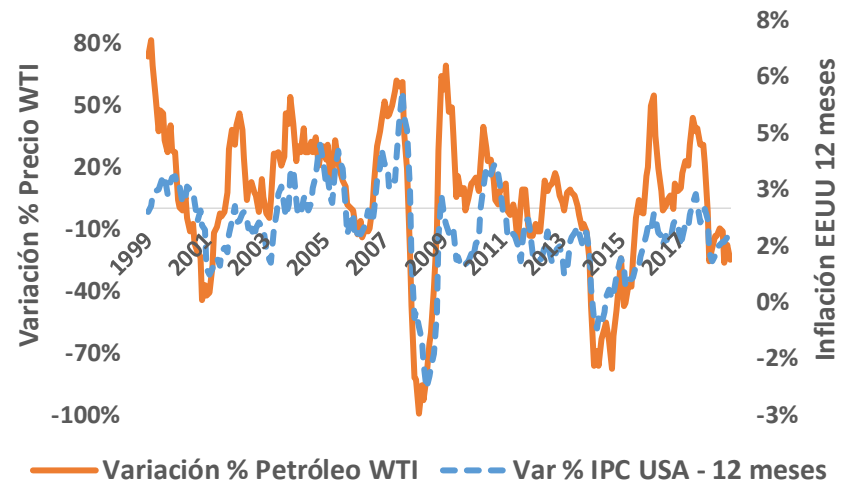

Fuente: Elaboración propia, datos FRED de ST Louis y Bloomberg.

Acudiendo al contexto anterior, se procedió a realizar los test de raíz unitaria para intercepto y tendencia de las variables de modelo. Como resultado se encuentra evidencia estadística de no estacionariedad en la razón de precios del petróleo WTI y la TRM (ver tablas 3, 4, 5 y 6 al final). En consecuencia, se propone entonces que la especificación del modelo está dada por la siguiente expresión general de la representación VEC:

$$
\left[\begin{array}{c}
\Delta \ln \left(E_{t}\right) \\
\Delta \ln \left(W T I_{t}\right) \\
\Delta \ln \left(P_{t}\right)
\end{array}\right]=\mathrm{A}_{0}+\sum_{i=1}^{J} \mathrm{~B}_{i}\left[\begin{array}{c}
\Delta \ln \left(E_{t-i}\right) \\
\Delta \ln \left(W T I_{t-i}\right) \\
\Delta \ln \left(P_{t-i}\right)
\end{array}\right]-\Pi\left[\begin{array}{c}
\ln \left(E_{t-1}\right) \\
\ln \left(W T I_{t-1}\right) \\
\ln \left(P_{t-1}\right)
\end{array}\right]+\left[\begin{array}{c}
u_{E, t} \\
u_{W T I, t} \\
u_{P, t}
\end{array}\right]
$$

donde:

P: el IPC colombiano dividido entre el IPC estadounidense a precios base de un mismo año y la frecuencia está en meses.

E: la tasa de cambio pesos por dólar.

WTI: el precio del petróleo WTI.

La magnitud del retardo "J", en la ecuación planteada, se escogió por el criterio de Akaike (1974) (ver Tabla 7).

$$
\begin{aligned}
& \Leftrightarrow \Delta Y_{t}=\mathrm{A}_{0}+\sum_{i=1}^{J} \mathrm{~B}_{i} \Delta Y_{t-i}-\Pi Y_{t-1}+u_{t} \forall u_{t} \sim(0, \Omega) ; \Delta Y_{t} \sim I(0) . \\
& \text { donde: } \Pi=\mathrm{I}-C \Lambda C^{-1} \Leftrightarrow \Pi=C[\mathrm{I}-\Lambda] C^{-1} \forall \Lambda=\left[\begin{array}{ccc}
\lambda_{E} & 0 & 0 \\
0 & \lambda_{W T I} & 0 \\
0 & 0 & \lambda_{P}
\end{array}\right] \\
& \Rightarrow \Pi=C[\mathrm{I}-\Lambda] C^{-1} \Leftrightarrow \Pi=C\left[\begin{array}{ccc}
1-\lambda_{E} & 0 & 0 \\
0 & 1-\lambda_{W T I} & 0 \\
0 & 0 & 1-\lambda_{P}
\end{array}\right] C^{-1} \Leftrightarrow \Pi=\alpha^{\prime} \beta
\end{aligned}
$$


Donde " $\alpha$ " es la matriz de velocidad de ajuste y " $\beta$ " es la matriz de coeficientes que forman la combinación lineal que cointegra a las variables y " $\lambda_{k}$ " denota las raíces características de cada variable. De esto se concluye que el efecto de las raíces unitarias se desvanece (en el caso que existan raíces unitarias), lo que deja únicamente las combinaciones lineales que son estacionarias. Teniendo en cuenta lo anterior y los métodos de Johansen (1988) y Johansen y Juselius (1990), se realiza el test de traza (ver Tabla 1), donde se prueba el rango de la matriz " $\Pi$ ", que indica la cantidad de vectores cointegradores e identifica la estructura del modelo.

\section{Pronóstico.}

Una vez se rechaza la hipótesis de no cointegración y habiendo identificado la estructura del modelo por el test de traza mencionado con anterioridad, se puede proceder a solucionar la ecuación dinámica contenida en el modelo VEC mediante la siguiente lógica:

$$
\begin{aligned}
& \Delta Y_{t}=\mathrm{A}_{0}+\sum_{i=1}^{J} \mathrm{~B}_{i} \Delta Y_{t-i}-\Pi Y_{t-1}+u_{t} \\
& \Rightarrow \Delta Y_{t}=\mathrm{B}(\mathrm{L})^{-1} \mathrm{~A}_{0}-\mathrm{B}(\mathrm{L})^{-1} \Pi Y_{t-1}+\mathrm{B}(\mathrm{L})^{-1} u_{t} \forall \mathrm{B}(\mathrm{L})^{-1}=\left(\mathrm{I}-\sum_{i=1}^{j} \mathrm{~B}_{i}\right)^{-1} \text {, de donde se }
\end{aligned}
$$
deduce que:

$$
\Delta Y_{t} \sim I(0) \Leftrightarrow\left(\mathrm{B}(\mathrm{L})^{-1} \mathrm{~A}_{0}-\mathrm{B}(\mathrm{L})^{-1} \Pi Y_{t-1}+\mathrm{B}(\mathrm{L})^{-1} u_{t}\right) \sim I(0)
$$

De esto se puede ver que:

$$
\begin{aligned}
& Y_{t}=\mathrm{B}(\mathrm{L})^{-1} \mathrm{~A}_{0}+\left(\mathrm{I}-\mathrm{B}(\mathrm{L})^{-1} \Pi\right) Y_{t-1}+\mathrm{B}(\mathrm{L})^{-1} u_{t} \text {, donde por iteración se encuentra que: } \\
& \mathrm{Z}_{t+k}=\left(\mathrm{I}-\mathrm{B}(\mathrm{L})^{-1} \Pi\right)^{k} \mathrm{Z}_{\mathrm{t}}+\left(\mathrm{I}-\mathrm{B}(\mathrm{L})^{-1} \Pi\right)^{k-1} u_{\mathrm{t}+1}+\cdots+u_{t+k} \forall \mathrm{Z}_{t}=Y_{t}-\mu_{Y}
\end{aligned}
$$

De modo que $E\left[\mathrm{Z}_{t+k}\right]=\left(\mathrm{I}-\mathrm{B}(\mathrm{L})^{-1} \Pi\right)^{k} \mathrm{Z}_{\mathrm{t}}$ gobernará el pronóstico para el horizonte deseado.

En cuanto a la constante se puede probar, por método iterativo, que ésta obedece a la siguiente expresión:

$$
\mu_{t+k}=\sum_{k=1}^{N}\left(\mathrm{I}-\mathrm{B}(\mathrm{L})^{-1} \Pi\right)^{k-1} \mathrm{~B}(\mathrm{~L})^{-1} \mathrm{~A}_{0}
$$

Por otra parte, los errores de pronostico denominados "e" están dados por:

$$
\mathrm{e}_{t+k}=\mathrm{Z}_{t+k}-E\left[\mathrm{Z}_{t+k}\right] \Leftrightarrow \mathrm{e}_{t+k}=\left(\mathrm{I}-\mathrm{B}(\mathrm{L})^{-1} \Pi\right)^{k-1} u_{t+1}+\cdots+u_{t+k}
$$

De modo que su varianza va estar especificada por " $\Sigma=E\left[\mathrm{ee}^{\prime}\right]$ " lo que para el " $\mathrm{t}+\mathrm{k}$ "-ésimo periodo se expresa como:

$$
\begin{aligned}
& \Sigma_{t+k}=\Omega+\Psi \Omega \Psi^{\prime}+\Psi^{2} \Omega\left(\Psi^{\prime}\right)^{2}+\cdots+\Psi^{k-1} \Omega\left(\Psi^{\prime}\right)^{k-1} \\
& \forall \Psi=\left(\mathrm{I}-\mathrm{B}(\mathrm{L})^{-1} \Pi\right) ; \Omega=E\left[u u^{\prime}\right] ; \Sigma_{t+k}=\left[\begin{array}{ccc}
\sigma_{E, t+k}^{2} & \sigma_{E, W T I, t+k} & \sigma_{E, P, t+k} \\
\sigma_{W T I, E, t+k} & \sigma_{W T I, t+k}^{2} & \sigma_{W T I, P, t+k} \\
\sigma_{P, E, t+k} & \sigma_{P, W T I, t+k} & \sigma_{P, t+k}^{2}
\end{array}\right]
\end{aligned}
$$

donde " $\Sigma_{k}$ " contiene las varianzas de cada error que gobernarán la incertidumbre alrededor del pronóstico en " $\mathrm{t}+\mathrm{k}$ " periodos en el futuro. 


\section{Resultados de la estimación.}

El cálculo del modelo se realizó con datos a partir de junio del 2008, momento para el que habían ya transcurrido ocho años desde la implementación del régimen cambiario flexible a finales de los 90 (Urrutia \& Llano, 2012), esto se hizo con la intención de mejorar la bondad de ajuste y hacer más certeros los pronósticos.

Tras realizar el Test de Traza por los métodos Johansen (1988) y Johansen y Juselius (1990), se concluyó que el modelo que mejor se ajusta a los datos es uno con constante en la expresión VEC y con constante en la ecuación de cointegración (Tabla 1). De ahí se propone que solo existe una ecuación de cointegración " $f(E, W T I, P)$ " de la forma:

$$
f(E, W T I, P)_{t}=\ln \left(E_{t}\right)-1.51 \ln \left(P_{t}\right)+0.52 \ln \left(W T I_{t}\right)-9.3(\text { ver Tabla 2). }
$$

Que es estacionaria con valor esperado " $E\left[f(E, W T I, P)_{t}\right]=0$ ", por lo tanto, cualquier choque exógeno que saque a las variables del equilibrio estocástico dinámico se desvanecerá gradualmente, a medida que las variables que forman la función se van ajustando. Como ejemplo se puede observar el Gráfico 5, que muestra la relación de cointegración y como ésta retorna al equilibrio a partir de agosto del 2008 y se mantiene ahí desde finales del 2008 hasta la actualidad.

\section{Gráfico 5. Relación de Cointegración.}

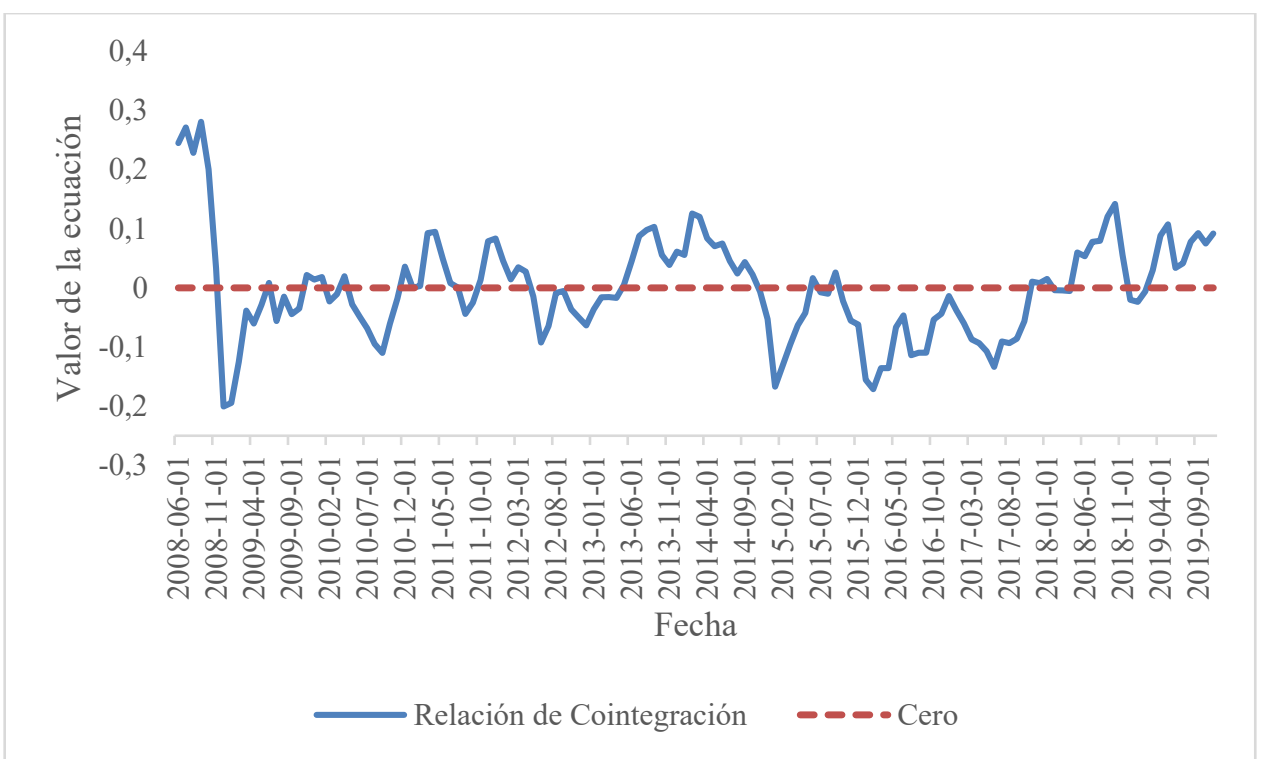

Fuente: Elaboración propia con Eviews a partir de los datos de la FRED de ST Louis, Banco de la República de Colombia (Banco de la Republica de Colombia, 2019) y el DANE.

Según el vector de velocidad de ajuste " $\boldsymbol{\alpha}$ " " la calibración promedio de las variables, a un $1 \%$ de desviación del equilibrio, es de $-0,065 \%$ para la tasa de cambio, $-0,45 \%$ para el WTI y $-0,005 \%$ para la razón de precios, donde esta última no es significativa al 5\% de significancia estadística, pero si lo es al 10\%. Esto indica que el WTI es la variable que menos demora en ajustarse. En cuanto a los precios, su coeficiente de velocidad muestra que estos presentan rigideces en el corto plazo, lo que unido a la significancia que tienen dentro de la ecuación de cointegración, permite suponer que su ajuste es una propiedad de largo plazo. 
En otras cosas los efectos cruzados de las variables mostraron que el primer retardo en el cambio logarítmico de la tasa de cambio impacta de manera significativa al primer cambio en el logaritmo de todas las variables a excepción del WTI. Lo mismo evidenció el segundo retardo lo que indica que no existe evidencia de relación alguna, por lo menos en los dos primeros retardos, entre la tasa de cambio pesos por dólar y el WTI. Lo anterior afirma entonces la hipótesis hecha previamente, en la que se proponía que el petróleo podía responder a la tasa de cambio, que reflejaba los movimientos del dólar a nivel mundial en el largo plazo.

Lo anterior responde a que, en el corto plazo, al menos en los dos primeros retardos, parece no haber relación alguna, lo que tiene sentido porque la producción de petróleo no puede expandirse a menos que se adapte rápidamente el capital productivo, y esto es muy difícil debido a la especificidad técnica que requiere. Por el contrario, en el largo plazo sí existe la posibilidad de adaptar el capital y la producción petrolera mundial y eso se evidencia en el coeficiente positivo dentro de la relación de cointegración, que explica que, para mantener la ecuación balanceada, el WTI tiene que moverse en forma inversa a los movimientos del dólar en el largo plazo.

En la razón de precios se encontró evidencia de que el cambio logarítmico del primer retardo solo impacta de manera significativa al cambio logarítmico de la tasa de cambio y al de la razón de precios, lo que es congruente con la hipótesis PPP. Respecto al segundo retardo no se encontró relación con ninguna variable bajo el criterio de que todos los estadísticos " $t$ " sean mayores que 1,1 .

Por último, el primer retardo del cambio logarítmico del WTI impactó de manera significativa al cambio logarítmico de la razón de precios y al cambio logarítmico del mismo WTI. Esto último confirma el hecho de que el combustible juega un rol en la inflación relativa. También es relevante aclarar que el $\mathrm{R}^{2}$ del modelo fue de $21,7 \%$.

\subsection{Análisis de impulso respuesta.}

En el análisis de impulso respuesta (Gráfico 5.1), la respuesta del WTI a la tasa de cambio refuerza la hipótesis que se había planteado anteriormente donde se proponía que la respuesta óptima de los productores de petróleo al precio del dólar a nivel internacional, reflejado en la tasa de cambio de Colombia, podía hacer que el precio del WTI se moviera de manera inversa debido a los incentivos que el precio del dólar produce en la producción de petróleo en el largo plazo.

También se observa que el impacto de la razón de precios sobre el WTI es negativo en el corto plazo y permanentemente positiva en el largo plazo. Este fenómeno no tiene base teórica dentro de la hipótesis de paridad por poder adquisitivo y queda abierta la posibilidad para su investigación en otros trabajos, en vista de que no es la intención del trabajo presente hacerlo. Respecto a sí mismo el impacto del WTI tiende a estabilizarse en cero en el largo plazo debido a que éste es estacionario en la muestra extraída (ver Tablas 3-6).

En cuanto al impacto de las variables sobre la razón de precios, todas muestran tener magnitudes positivas. El efecto del WTI sobre la razón de precios se debe al efecto que tiene el incremento de precios de la energía en la cesta básica (ya mencionado con anterioridad), mientras que lo mismo puede afirmarse de la tasa de cambio sobre la cesta de bienes importados, aunque la respuesta al WTI para la muestra extraída no muestra ser significativamente diferente de cero a dos errores estándar. Por último, la respuesta de la razón de precios a sí misma muestra ser permanente; esto se debe a que esta variable no es estacionaria.

Respecto a las respuestas de la tasa de cambio a sí misma y las otras variables se nota que responde a sí misma y al WTI de manera positiva en el largo plazo y de forma negativa a la razón de precios, lo que contradice la hipótesis PPP y a la ecuación de largo plazo que cointegra las variables estimada por el modelo (Tabla 2). Lo anterior no es un problema si se tiene en cuenta que a 2 desviaciones estándar como se ve en el Gráfico 5.1 el impulso respuesta de la tasa de cambio al WTI y a la razón de precios no muestra ser significativamente diferente de cero. 
Es preciso aclarar que de los impulsos respuesta analizados anteriormente únicamente dos fueron estadísticamente diferentes de cero y corresponden a las respuestas de la razón de precios y el WTI a la tasa de cambio, lo que podría estar sugiriendo en un principio que la tasa de cambio es el causante en cómo se mueven estas dos variables, y no al contrario, lo que no supone contradicción alguna con la hipótesis paridad débil de poder adquisitivo, ya que ésta solo propone que las variables involucradas se ajustan para mantenerse equilibradas.

\section{Gráfico 5.1 Análisis de impulso respuesta.}

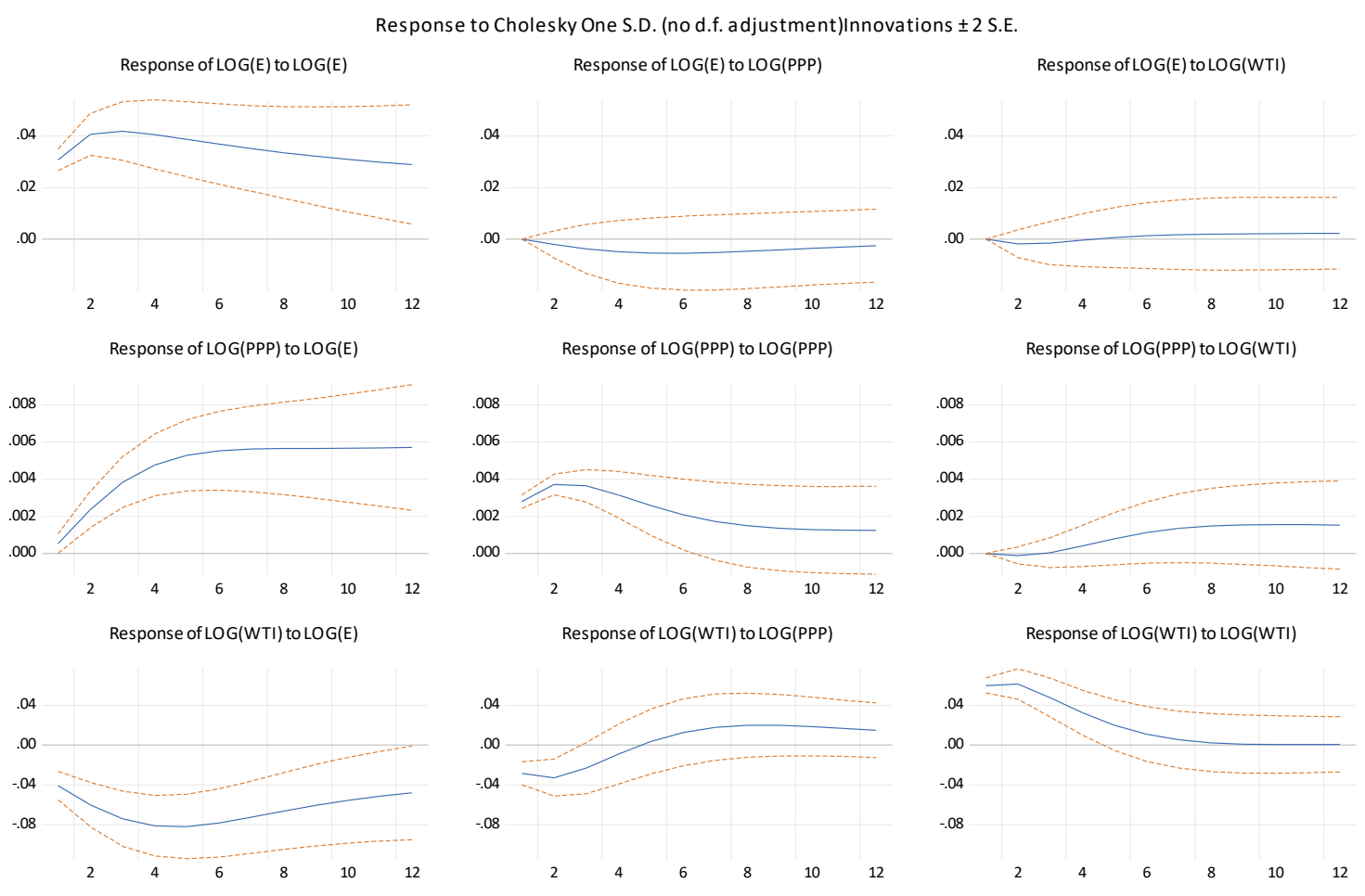

Fuente: Elaboración propia con Eviews a partir de los datos de la FRED de ST Louis, Banco de la República de Colombia (Banco de la Republica de Colombia, 2019) y el DANE.

\subsection{Test de Causalidad de Granger.}

El resultado de este test (Tabla 8) dista un poco de los resultados vistos en el Gráfico 5.1, ya que según el test de causalidad de Granger (Granger, 1969), de manera individual, las únicas variables que causan a otras en el sentido de Granger al 5\% de significancia estadística, son la tasa de cambio hacia la razón de precios y el WTI hacia la tasa de cambio, donde este último aparecía como un efecto no significativo según el análisis de impulso respuesta.

No obstante, lo descrito en el párrafo anterior, como se ve en la tabla 8 , el test conjunto arroja que todas las variables causan a las otras en el sentido de Granger con la única excepción del WTI, en vista de que ninguna de las variables lo causa ni de forma individual como conjunta. Esto unido al descarte de la magnitud del impacto en los impulsos respuesta no significativos, y aceptando la significancia en la ecuación de largo plazo, nos permite hacernos a la idea de que es correcto pronosticar la tasa de cambio para Colombia teniendo en cuenta los efectos conjuntos del WTI y la razón de precios.

\subsection{Resultados del pronóstico.}

Tras resolver la ecuación dinámica del VEC se encontró que la tasa de cambio tiene una senda ascendente en su valor esperado, con una varianza creciente en el tiempo. Los resultados arrojan que se 
espera una tasa de cambio de $\$ 3.436$ para diciembre de 2019, $\$ 3685$ para junio del 2020 y $\$ 3789$ para diciembre del 2020; estos resultados están representados en los siguientes gráficos con errores de pronóstico de dos desviaciones estándar.

\section{Gráfico 6. Resultados del pronóstico para la tasa de cambio del peso colombiano contra el dólar estadounidense (USD/COP).}

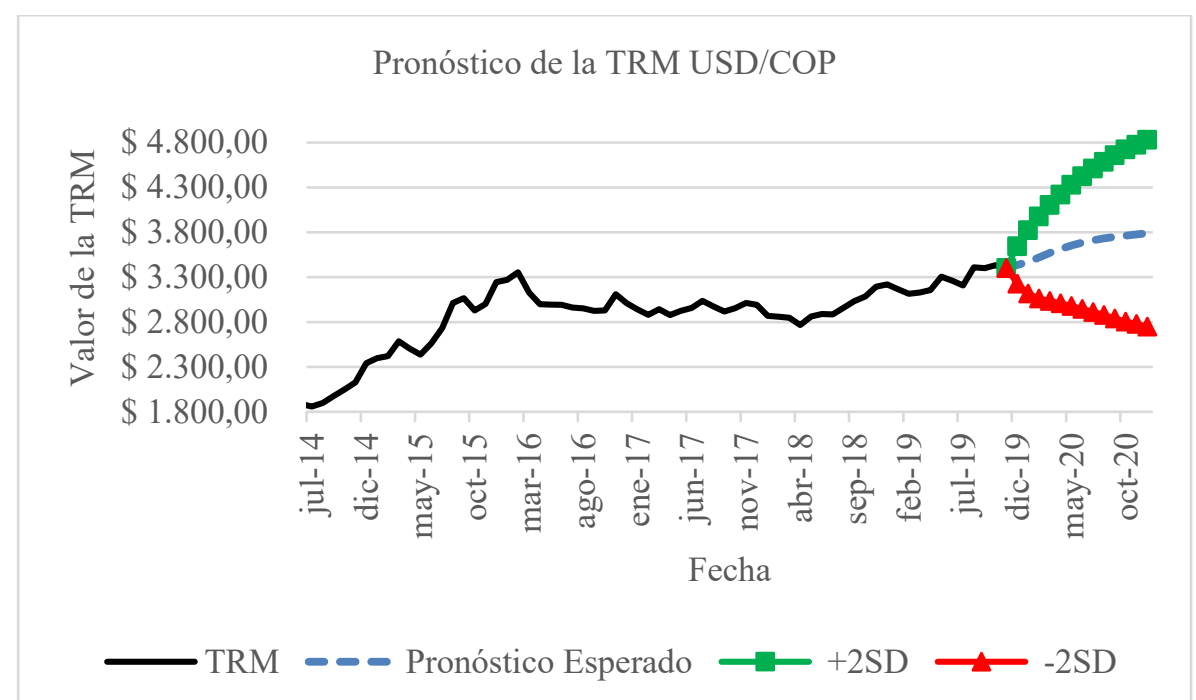

Fuente: Elaboración propia con Eviews a partir de los datos de la FRED de ST Louis, Banco de la República de Colombia y el DANE.

Gráfico 7. Resultados del pronóstico para la razón de precios.

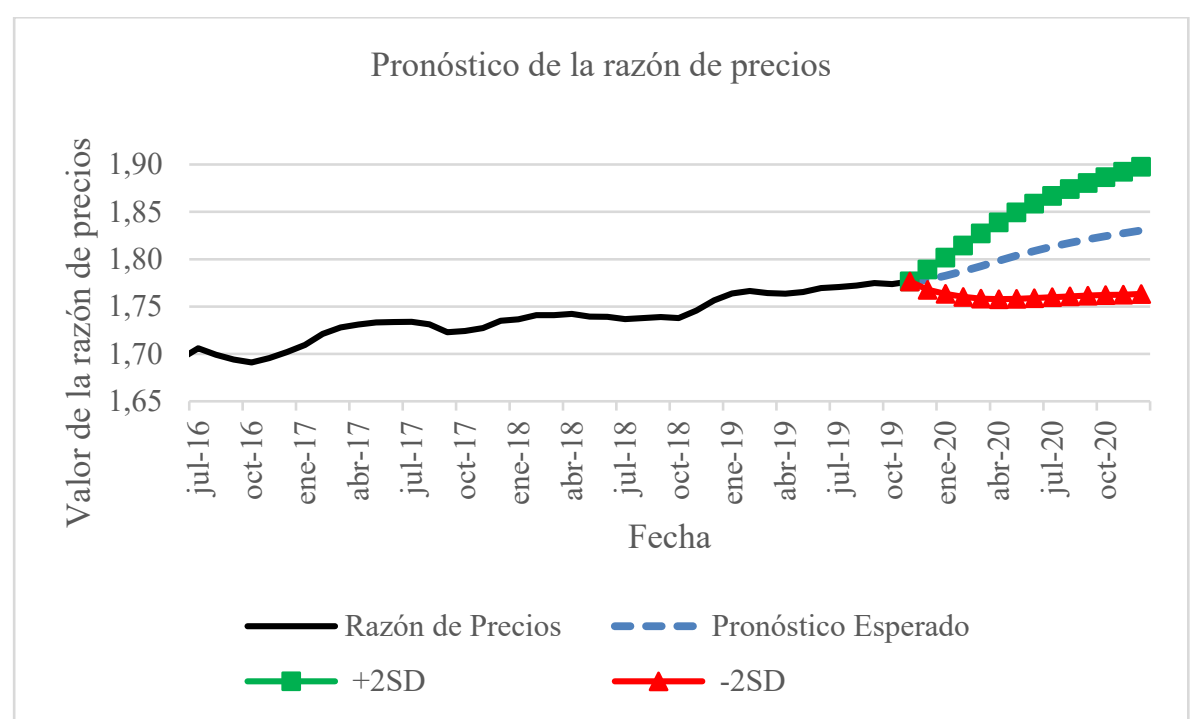

Fuente: Elaboración propia con Eviews a partir de los datos de la FRED de ST Louis, Banco de la República de Colombia y el DANE. 
Gráfico 8. Resultados del pronóstico para el precio del WTI.

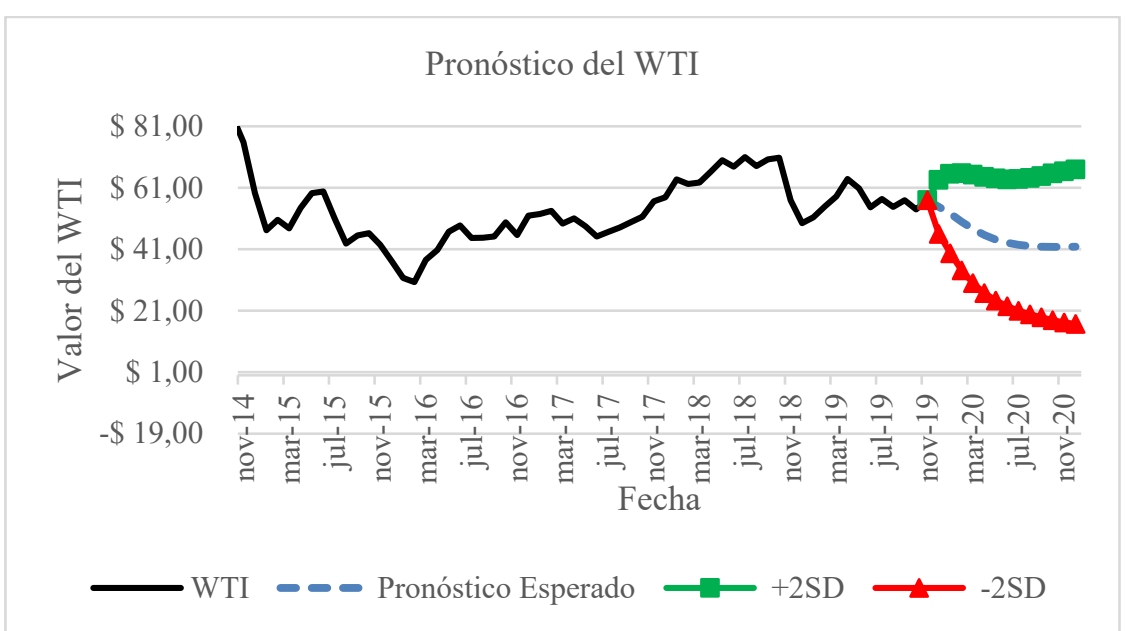

Fuente: Elaboración propia con Eviews a partir de los datos de la FRED de ST Louis, Banco de la República de Colombia y el DANE.

\section{Conclusiones.}

Para este caso la hipótesis de cointegración entre las variables propuestas se cumple y aunque los precios parecen presentar rigideces en el corto plazo, debido a que la velocidad de ajuste en la razón de los mismos no es significativa, en el largo plazo sí existe evidencia de ajuste según lo indica la ecuación de cointegración. Se puede concluir que los efectos del índice dólar, reflejados en la tasa de cambio colombiana, impacta, con excepción del WTI, como cambian las variables dos meses en el futuro, mientras lo mismo ocurre con el cambio logarítmico de la razón de precios un mes a futuro. Por su lado, el cambio logarítmico del petróleo, solo tiene impacto sobre sí mismo y la razón de precios un mes en el futuro, lo que confirma la hipótesis planteada en cuanto al efecto del petróleo sobre los precios. Teniendo en cuenta al segundo retardo, la evidencia mostró que el cambio logarítmico del petróleo se impacta a sí mismo y al cambio logarítmico del dólar (Tabla 2). Todo esto confirma la hipótesis débil de PPP y la incidencia del WTI sobre la tasa de cambio pesos por dólar.

Los test de raíz unitaria para intercepto y tendencia de las variables de modelo, dieron evidencia estadística de no estacionariedad en la razón de precios y la TRM, hecho que validó la primera fase de la investigación.

Muchos de los efectos impulso respuesta no fueron significativos ya que a dos errores estándar se encontraba espacio por encima y debajo de cero.

Una gran cantidad de los efectos significativos a corto plazo dados por el efecto de las variables a 1 o 2 retardos, fueron de menor magnitud que el efecto que había en la ecuación de largo plazo, lo que quiere decir que justamente prima el efecto de la ecuación de largo plazo.

El Test de causalidad de Granger, aunque individualmente da pocas causalidades en el sentido de Granger, en el sentido conjunto prueba causalidad que es justamente lo que, sumado a las demás pruebas realizadas, da la validez científica necesaria para realizar pronósticos con el modelo VEC propuesto.

Según el modelo el pronóstico para la tasa de cambio USD/COP, dado el comportamiento de la razón de precios y el WTI, indica que la tasa de cambio terminaría alrededor de los $\$ 3436$ en diciembre de 2019 y en $\$ 3789$ para diciembre del 2020. 
Para finalizar, al mismo tiempo se obtuvo el pronóstico para el precio del WTI, basado en el modelo de tasa de cambio, que mantiene en evidencia la relación inversa entre la cotización del petróleo y la tasa de cambio USD/COP al mostrar niveles cercanos a los \$41 dólares para el año 2020.

\section{Anexo de Tablas Estadísticas.}

Tabla 1. Test de Traza por el método de Johansen y Juselius, Maximum likelihood estimation and inference on cointegration.

Date: 12/13/19 Time: 21:01

Sample (adjusted): 2008M06 2019M11

Included observations: 138 after adjustments

Trend assumption: Linear deterministic trend

Series: LOG(E) LOG(PPP) LOG(WTI)

Lags interval (in first differences): 1 to 2

\begin{tabular}{ccccc}
\hline \hline \multicolumn{5}{c}{ Unrestricted Cointegration Rank Test (Trace) } \\
\hline \hline $\begin{array}{c}\text { Hypothesized } \\
\text { No. of CE(s) }\end{array}$ & Eigenvalue & $\begin{array}{c}\text { Trace } \\
\text { Statistic }\end{array}$ & $\begin{array}{c}0.05 \\
\text { Critical Value Prob.** }\end{array}$ \\
\hline \hline None * & 0.175409 & 35.81337 & 29.79707 & 0.0090 \\
At most 1 & 0.062493 & 9.197540 & 15.49471 & 0.3474 \\
At most 2 & 0.002115 & 0.292236 & 3.841466 & 0.5888 \\
\hline \hline
\end{tabular}

Trace test indicates 1 cointegrating eqn(s) at the 0.05 level

$*$ denotes rejection of the hypothesis at the 0.05 level

**MacKinnon-Haug-Michelis (1999) p-values

Unrestricted Cointegration Rank Test (Maximum Eigenvalue)

\begin{tabular}{ccccc}
\hline \hline $\begin{array}{c}\text { Hypothesized } \\
\text { No. of CE(s) }\end{array}$ & Eigenvalue & $\begin{array}{c}\text { Max-Eigen } \\
\text { Statistic }\end{array}$ & $\begin{array}{c}0.05 \\
\text { Critical Value Prob.** }\end{array}$ \\
\hline \hline None $*$ & 0.175409 & 26.61583 & 21.13162 & 0.0076 \\
At most 1 & 0.062493 & 8.905304 & 14.26460 & 0.2941 \\
At most 2 & 0.002115 & 0.292236 & 3.841466 & 0.5888 \\
\hline \hline
\end{tabular}

Max-eigenvalue test indicates 1 cointegrating eqn(s) at the 0.05 level

$*$ denotes rejection of the hypothesis at the 0.05 level

**MacKinnon-Haug-Michelis (1999) p-values

Unrestricted Cointegrating Coefficients (normalized by $b^{\prime * S} 11^{*} b=I$ ):

\begin{tabular}{ccc}
\hline \hline LOG(E) & LOG(PPP) & LOG(WTI) \\
12.97756 & -19.68354 & 6.852804 \\
-13.19937 & 36.20136 & -0.360587 \\
4.962814 & 4.780093 & 1.896705 \\
\hline \hline
\end{tabular}

Unrestricted Adjustment Coefficients (alpha):

\begin{tabular}{cccc}
\hline \hline $\mathrm{D}(\mathrm{LOG}(\mathrm{E}))$ & 0.005060 & 0.000537 & 0.001236 \\
$\mathrm{D}(\mathrm{LOG}(\mathrm{PPP}))$ & 0.000419 & -0.000676 & $2.57 \mathrm{E}-05$ \\
$\mathrm{D}(\mathrm{LOG}(\mathrm{WTI}))$ & -0.034807 & 0.003084 & -0.000572 \\
\hline \hline
\end{tabular}

1 Cointegrating Equation(s): Log likelihood 1098.103

Normalized cointegrating coefficients (standard error in parentheses) 


$\begin{array}{ccc}\text { LOG(E) } & \text { LOG(PPP) } & \text { LOG(WTI) } \\ 1.000000 & -1.516737 & 0.528050 \\ & (0.27413) & (0.06833)\end{array}$

Adjustment coefficients (standard error in parentheses)

$\begin{array}{cc}\mathrm{D}(\text { LOG(E)) } & 0.065670 \\ & (0.03313) \\ \mathrm{D}(\text { LOG(PPP) }) & 0.005432 \\ & (0.00331) \\ \mathrm{D}(\text { LOG(WTI)) } & -0.451712 \\ & (0.08818)\end{array}$

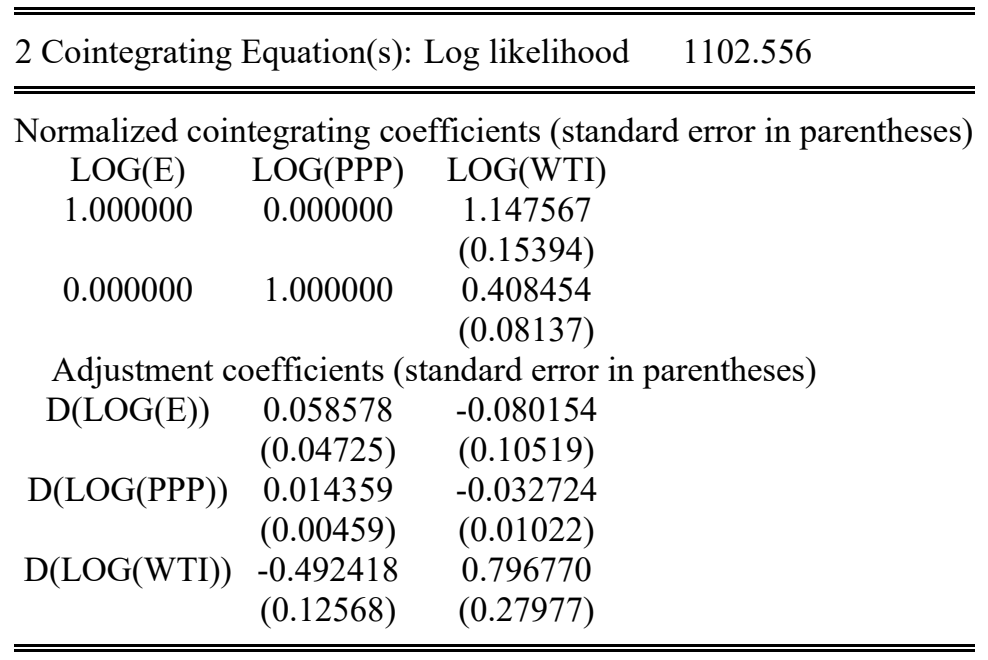

Fuente: Elaboración propia con Eviews a partir de los datos de la FRED de ST Louis, El Banco de la República de Colombia y el DANE.

Tabla 2. Resultado del cálculo del Modelo VEC.

Vector Error Correction Estimates

Date: 12/13/19 Time: 13:50

Sample (adjusted): 2008M06 2019M11

Included observations: 138 after adjustments

Standard errors in ( ) \& t-statistics in [ ]

\begin{tabular}{cccc}
\hline \hline Cointegrating Eq: & CointEq1 & & \\
\hline \hline LOG(E(-1)) & 1.000000 & & \\
LOG(PPP(-1)) & -1.516737 & & \\
& $(0.27413)$ & & \\
& {$[-5.53298]$} & & \\
LOG(WTI(-1)) & 0.528050 & & \\
& $(0.06833)$ & & \\
& {$[7.72831]$} & & \\
C & -9.302999 & & \\
& & & \\
\hline \hline \multirow{2}{*}{ Error Correction: } & D(LOG(E)) & D(LOG(PPP)) $(\mathrm{LOG}(\mathrm{WTI}))$ \\
\hline \hline & & & \\
CointEq1 & 0.065670 & 0.005432 & -0.451712 \\
& $(0.03313)$ & $(0.00331)$ & $(0.08818)$ \\
D(LOG(E(-1))) & {$[1.98199]$} & {$[1.64114]$} & {$[-5.12261]$} \\
& 0.291733 & 0.040050 & -0.100445 \\
& $(0.09796)$ & $(0.00979)$ & $(0.26070)$ \\
D(LOG(E(-2))) & {$[2.97816]$} & {$[4.09292]$} & {$[-0.38529]$} \\
& -0.275120 & 0.022481 & 0.051473 \\
& $(0.10583)$ & $(0.01057)$ & $(0.28166)$
\end{tabular}




\begin{tabular}{|c|c|c|c|}
\hline \multirow{3}{*}{ D(LOG(PPP(-1))) } & {$[-2.59961]$} & {$[2.12658]$} & [ 0.18275$]$ \\
\hline & -1.351410 & 0.344505 & -0.709152 \\
\hline & $(0.97594)$ & $(0.09749)$ & $(2.59734)$ \\
\hline & {$[-1.38473]$} & [ 3.53381$]$ & {$[-0.27303]$} \\
\hline \multirow[t]{3}{*}{$\mathrm{D}(\mathrm{LOG}(\mathrm{PPP}(-2)))$} & -0.107607 & 0.064894 & 0.072870 \\
\hline & $(0.85913)$ & $(0.08582)$ & $(2.28646)$ \\
\hline & {$[-0.12525]$} & {$[0.75617]$} & [ 0.03187$]$ \\
\hline \multirow[t]{3}{*}{ D(LOG(WTI(-1))) } & -0.034457 & -0.006448 & 0.275066 \\
\hline & $(0.03881)$ & $(0.00388)$ & $(0.10329)$ \\
\hline & {$[-0.88778]$} & {$[-1.66313]$} & [2.66292] \\
\hline \multirow[t]{3}{*}{ D(LOG(WTI(-2))) } & -0.131393 & 0.004201 & 0.190854 \\
\hline & $(0.03953)$ & $(0.00395)$ & $(0.10521)$ \\
\hline & {$[-3.32357]$} & [ 1.06386$]$ & [ 1.81397$]$ \\
\hline \multirow[t]{3}{*}{$\mathrm{C}$} & 0.006394 & 0.000754 & -0.002022 \\
\hline & $(0.00304)$ & $(0.00030)$ & $(0.00809)$ \\
\hline & [2.10363] & [2.48242] & {$[-0.25000]$} \\
\hline R-squared & 0.217307 & 0.522676 & 0.302990 \\
\hline Adj. R-squared & 0.175162 & 0.496973 & 0.265459 \\
\hline Sum sq. resids & 0.116941 & 0.001167 & 0.828280 \\
\hline S.E. equation & 0.029992 & 0.002996 & 0.079821 \\
\hline F-statistic & 5.156173 & 20.33592 & 8.073008 \\
\hline Log likelihood & 292.2471 & 610.1535 & 157.1669 \\
\hline Akaike AIC & -4.119524 & -8.726862 & -2.161839 \\
\hline Schwarz SC & -3.949828 & -8.557166 & -1.992143 \\
\hline Mean dependent & 0.004712 & 0.001788 & -0.005717 \\
\hline S.D. dependent & 0.033024 & 0.004224 & 0.093134 \\
\hline \multicolumn{2}{|c|}{ Determinant resid covariance (dof adj.) } & $2.94 \mathrm{E}-11$ & \\
\hline \multicolumn{2}{|c|}{ Determinant resid covariance } & $2.46 \mathrm{E}-11$ & \\
\hline \multicolumn{2}{|c|}{ Log likelihood } & 1098.103 & \\
\hline \multicolumn{2}{|c|}{ Akaike information criterion } & -15.52323 & \\
\hline \multicolumn{2}{|c|}{ Schwarz criterion } & -14.95051 & \\
\hline \multicolumn{2}{|c|}{ Number of coefficients } & 27 & \\
\hline
\end{tabular}

Fuente: Elaboración propia con Eviews a partir de los datos de la FRED de ST Louis, El Banco de la República de Colombia y el DANE.

Tabla 3. Test de raíz unitaria para intercepto y tendencia TRM Banco de la República de Colombia.

Null Hypothesis: E has a unit root

Exogenous: Constant, Linear Trend

Lag Length: 1 (Automatic - based on SIC, maxlag=13)

\begin{tabular}{cc}
\hline \hline & t-Statistic Prob.* \\
\hline \hline Augmented Dickey-Fuller test statistic & -1.9125160 .6426 \\
\hline Test critical values: $1 \%$ level & -4.026942 \\
$5 \%$ level & -3.443201 \\
$10 \%$ level & -3.146309 \\
\hline \hline *MacKinnon (1996) one-sided p-values. \\
Augmented Dickey-Fuller Test Equation \\
Dependent Variable: D(E) \\
Method: Least Squares \\
Sample (adjusted): 2008M08 2019M11 \\
Included observations: 136 after adjustments \\
\hline \hline Variable CoefficientStd. Error t-Statistic Prob.
\end{tabular}




\begin{tabular}{crrrr}
\hline \hline $\mathrm{E}(-1)$ & -0.040669 & 0.021265 & -1.9125160 .0580 \\
$\mathrm{D}(\mathrm{E}(-1))$ & 0.273340 & 0.083910 & 3.257543 & 0.0014 \\
$\mathrm{C}$ & 66.77959 & 36.56813 & 1.826169 & 0.0701 \\
@TREND("2008M06") & 0.557065 & 0.296957 & 1.875913 & 0.0629 \\
\hline \hline
\end{tabular}

Fuente: Elaboración propia con Eviews a partir de los datos de la FRED de ST Louis, El Banco de la República de Colombia y el DANE.

Tabla 4. Test de raíz unitaria intercepto y tendencia, Razón de índice de precios.

Null Hypothesis: PPP has a unit root

Exogenous: Constant, Linear Trend

Lag Length: 1 (Automatic - based on SIC, maxlag=13)

\begin{tabular}{ccl}
\hline \hline & t-Statistic Prob.* \\
\hline \hline \multicolumn{2}{c}{ Augmented Dickey-Fuller test statistic } & -1.8716480 .6637 \\
\hline Test critical values: & 1\% level & -4.026942 \\
& $5 \%$ level & -3.443201 \\
& $10 \%$ level & -3.146309 \\
\hline \hline
\end{tabular}

*MacKinnon (1996) one-sided p-values.

Augmented Dickey-Fuller Test Equation

Dependent Variable: D(PPP)

Method: Least Squares

Sample (adjusted): 2008M08 2019M11

Included observations: 136 after adjustments

\begin{tabular}{|c|c|}
\hline Variable & CoefficientStd. Error t-Statistic Prob. \\
\hline PPP(-1) & $-0.0224200 .011979-1.8716480 .0635$ \\
\hline $\mathrm{D}(\mathrm{PPP}(-1))$ & $\begin{array}{lll}0.578081 & 0.0709278 .150354 & 0.0000\end{array}$ \\
\hline $\mathrm{C}$ & 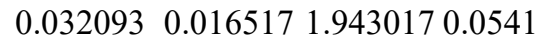 \\
\hline @TREND("2008M06") & $6.42 \mathrm{E}-05 \quad 3.60 \mathrm{E}-05 \quad 1.7827780 .0769$ \\
\hline
\end{tabular}

Fuente: Elaboración propia con Eviews a partir de los datos de la FRED de ST Louis, El Banco de la República de Colombia y el DANE.

Tabla 5. Test de raíz unitaria intercepto y tendencia, WTI.

Null Hypothesis: WTI has a unit root

Exogenous: Constant, Linear Trend

Lag Length: 1 (Automatic - based on SIC, maxlag=13)

\begin{tabular}{cc}
\hline \hline & t-Statistic Prob.* \\
\hline \hline Augmented Dickey-Fuller test statistic & -3.3631730 .0608 \\
\hline Test critical values: $\quad 1 \%$ level & -4.026942 \\
$5 \%$ level & -3.443201 \\
$10 \%$ level & -3.146309 \\
\hline \hline & \\
*MacKinnon (1996) one-sided p-values. \\
Augmented Dickey-Fuller Test Equation \\
Dependent Variable: D(WTI) \\
Method: Least Squares \\
Sample (adjusted): 2008M08 2019M11 \\
\hline \hline
\end{tabular}


Included observations: 136 after adjustments

\begin{tabular}{|c|c|}
\hline Variable & CoefficientStd. Error t-Statistic Prob. \\
\hline WTI(-1) & $-0.0831640 .024728-3.3631730 .0010$ \\
\hline $\mathrm{D}(\mathrm{WTI}(-1))$ & 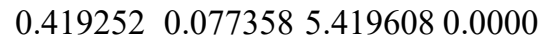 \\
\hline $\mathrm{C}$ & 6.6477722 .4786142 .6820530 .0083 \\
\hline @TREND("2008M06") & $-0.0135910 .014127-0.9620710 .3378$ \\
\hline
\end{tabular}

Fuente: Elaboración propia con Eviews a partir de los datos de la FRED de ST Louis, El Banco de la República de Colombia y el DANE.

Tabla 6. Test de raíz unitaria WTI con intercepto.

Null Hypothesis: WTI has a unit root

Exogenous: Constant

Lag Length: 1 (Automatic - based on SIC, maxlag=13)

\begin{tabular}{|c|c|c|}
\hline & & t-Statistic Prob.* \\
\hline \multicolumn{2}{|c|}{ Augmented Dickey-Fuller test statistic } & -3.3157810 .0160 \\
\hline \multirow[t]{3}{*}{ Test critical values: } & $1 \%$ level & -3.478911 \\
\hline & $5 \%$ level & -2.882748 \\
\hline & $10 \%$ level & -2.578158 \\
\hline \multicolumn{3}{|c|}{ *MacKinnon (1996) one-sided p-values. } \\
\hline \multicolumn{3}{|c|}{ Augmented Dickey-Fuller Test Equation } \\
\hline \multicolumn{3}{|c|}{ Dependent Variable: D(WTI) } \\
\hline \multicolumn{3}{|c|}{ Method: Least Squares } \\
\hline \multirow{2}{*}{\multicolumn{3}{|c|}{$\begin{array}{l}\text { Sample (adjusted): 2008M08 2019M11 } \\
\text { Included observations: } 136 \text { after adjustments }\end{array}$}} \\
\hline & & \\
\hline Variable & \multicolumn{2}{|c|}{ CoefficientStd. Error t-Statistic Prob. } \\
\hline WTI(-1) & \multicolumn{2}{|c|}{$-0.0715630 .021583-3.3157810 .0012$} \\
\hline D(WTI $(-1))$ & \multicolumn{2}{|c|}{0.4098180 .0767135 .3422330 .0000} \\
\hline $\mathrm{C}$ & \multicolumn{2}{|c|}{$4.858106 \quad 1.6375572 .9666780 .0036$} \\
\hline
\end{tabular}

Fuente: Elaboración propia con Eviews a partir de los datos de la FRED de ST Louis, El Banco de la República de Colombia y el DANE.

Tabla 7. Test de retardos óptimos en el VAR de la expresión VEC.

VAR Lag Order Selection Criteria

Endogenous variables: DLOG(E) DLOG(PPP) DLOG(WTI)

Exogenous variables:

Sample: 2008M06 2019M11

Included observations: 132

\begin{tabular}{ccccccc}
\hline \hline Lag & LogL & LR & FPE & AIC & SC & HQ \\
\hline \hline 1 & 1033.936 & NA & $3.62 \mathrm{e}-11$ & -15.52933 & $-15.33277^{*}-15.44946$ \\
2 & 1048.849 & $28.47104^{*}$ & $3.31 \mathrm{e}-11^{*}$ & $-15.61893^{*}$ & -15.22582 & $-15.45918^{*}$ \\
3 & 1055.611 & 12.60253 & $3.42 \mathrm{e}-11$ & -15.58502 & -14.99536 & -15.34541 \\
4 & 1060.309 & 8.540927 & $3.66 \mathrm{e}-11$ & -15.51983 & -14.73361 & -15.20035 \\
5 & 1069.535 & 16.35593 & $3.65 \mathrm{e}-11$ & -15.52326 & -14.54049 & -15.12391 \\
\hline \hline
\end{tabular}


* indicates lag order selected by the criterion

LR: sequential modified LR test statistic (each test at 5\% level)

FPE: Final prediction error

AIC: Akaike information criterion

SC: Schwarz information criterion

HQ: Hannan-Quinn information criterion

Fuente: Elaboración propia con Eviews a partir de los datos de la FRED de ST Louis, El Banco de la República de Colombia y el DANE.

Tabla 8. Test de causalidad de Granger.

VEC Granger Causality/Block Exogeneity Wald Tests Sample: 2008M06 2019M11

Included observations: 135

\begin{tabular}{cccc}
\hline \multicolumn{4}{c}{ Dependent variable: D(LOG(E)) } \\
\hline \hline Excluded & Chi-sq & df & Prob. \\
\hline \hline D(LOG(PPP)) & 2.260778 & 2 & 0.3229 \\
D(LOG(WTI)) & 12.76809 & 2 & 0.0017 \\
\hline \hline All & 14.29075 & 4 & 0.0064 \\
\hline \hline Dependent variable: D(LOG(PPP)) & \\
\hline \hline Excluded & Chi-sq & df & Prob. \\
\hline D(LOG(E)) & 22.23449 & 2 & 0.0000 \\
D(LOG(WTI)) & 3.244543 & 2 & 0.1974 \\
\hline \hline All & 36.57652 & 4 & 0.0000 \\
\hline \hline
\end{tabular}

Dependent variable: $\mathrm{D}(\mathrm{LOG}(\mathrm{WTI}))$

\begin{tabular}{cccc}
\hline \hline Excluded & Chi-sq & df & Prob. \\
\hline \hline D(LOG(E)) & 0.271428 & 2 & 0.8731 \\
D(LOG(PPP)) & 0.216362 & 2 & 0.8975 \\
\hline \hline All & 0.365579 & 4 & 0.9852 \\
\hline \hline
\end{tabular}

Fuente: Elaboración propia con Eviews a partir de los datos de la FRED de ST Louis, El Banco de la República de Colombia y el DANE.

\section{Referencias}

Akaike, H. (1974). A new look at the statistical model identification. IEEE Transactions on Automatic Control, 19(6), 716-723. https://doi.org/10.1109/TAC.1974.1100705

Amuzegar, J. (1978). OPEC and the Dollar Dilemma. Foreign Affairs, 56(4), 740-750.

Banco de la República de Colombia (2019, Diciembre 13). banrep.gov.co. Retrieved from https://www.banrep.gov.co/es/estadisticas/trm 
Cerquera, O.H., Gómez, C.F., \& Arias, C. J. (2018). Purchasing power parity in the Colombian exchange rate. Anfora, 25(45), 123-143. https://doi.org/10.30854/anf.v25.n45.2018.515.

Cochrane, S., \& DeFina, R. (1995). Predictable components in exchange rates. Quarterly Review of Economics and Finance, 35(1), 1-14.

Cumby, R., \& Obstfeld, M. (1984). International interest rate and price level linkage under flexible exchange rates: review of recent evidence. In R. M. Bilson, Exchange Rate Theory and Practice (pp. 121-151). Chicago, Estados Unidos: University of Chicago Press.

DANE (2019, agosto). DANE. Retrieved from https://www.dane.gov.co/index.php/estadisticas-portema/comercio-internacional/exportaciones

Engle, R.F., \& Granger, C. (1987). Co-integration and error correction: representation, estimation, and testing. Econometrica, 55(2), 251-267.

Federal Reserve Bank of St Louis (2019). fred.stlouisfed.org. Retrieved from https://fred.stlouisfed.org/series/TWEXB

Frenkel, J.A. (1981). Flexible Exchange Rates, Prices, and the Role of News: Lessons from the 1970s. Journal of Political Economy, 89(4), 665-705.

Garín, J., Lester, R., \& Sims, E. (2018). Intermediate Macroeconomics. Indiana: University of Notre Dame.

Granger, C.W. (1969). Investigating Causal Relations by Econometric Models and Cross-spectral Methods. Econometrica, 424-438.

Gregorio, J.D. (2012). POLICY CORNER: Commodity Prices, Monetary Policy, and Inflation. IMF Economic Review, 60, 600-633. https://doi.org/10.1057/imfer.2012.15

Hernández, C.F., Mesa, R.F., \& Pareja, I.V. (2009). Proyección de la tasa de cambio de Colombia bajo condiciones de PPA: evidencia empírica usando var. Estudios Gerenciales, 25(113), 211-226.

Holmes, M., \& Wang, P. (2005). Do African countries move asymmetrically towards Purchasing Power Parity? South African Journal of Economics, 73(2), 292-301. https://doi.org/10.1111/j.18136982.2005.00018.x

Jacobson, T., \& Nessen, M. (2004). Examining world-wide Purchasing Power Parity. Empirical Economics, 29, 463-476. https://doi.org/10.1007/s00181-003-0176-2

Johansen, S. (1988). Statistical analysis of cointegration vectors. Journal of Economic Dynamics and Control, 231-254. https://doi.org/10.1016/0165-1889(88)90041-3

Johansen, S., \& Juselius, K. (1990). Maximum likelihood estimation and inference on cointegration with applications to the demand for money. Oxford Bulletin of Economics and Statistics, 52(2), 169210. https://doi.org/10.1111/j.1468-0084.1990.mp52002003.x

Kim, Y. (1990). Purchasing power parity: another look at the long-run data. Economics Letters, 32(4), 339-344. DOI: https://doi.org/10.1016/0165-1765(90)90026-W

Krugman, P. (1978). Purchasing power parity and exchange rates. Journal of International Economics, 8(3), 397-407. DOI: https://doi.org/10.1016/0022-1996(78)90003-X 
Kugler, P., \& Lenz, C. (1993). Multivariate Cointegration Analysis and the Long-Run Validity of PPP. The Review of Economics and Statistics, 75(1), 180-184. https://doi.org/10.2307/2109645

Lipschitz, L., \& Schadler, S. (2019). Macroeconomics for Professionals: A guide for analysts and those who need to understand them. Cambridge, United Kingdom: Cambridge University Press.

Macdonald, R. (1993). The monetary approach to the exchange rate: rational expectations, long-run equilibrium, and forecasting. IMF Staff Papers, 92(34), 89-107.

Mankiw, N.G. (2016). Macroeconomics. Nueva York: Worth Publishers, Macmillan.

Papell, D., \& Podran, R. (2006). Additional Evidence of Long-Run Purchasing Power Parity with Restricted Structural Change. Journal of Money, Credit, and Banking, 38(5), 1329-1349. https://doi.org/10.1353/mcb.2006.0073.

Patel, J. (1990). Purchasing power parity as a long run relation. Journal of Applied Econometrics, 5(4), 367-379. https://doi.org/10.1002/jae.3950050405.

Serletis, A., \& Gogas, P. (2004). Long-horizon regression tests of the theory of purchasing power parity. Journal of Banking \& Finance, 28(8), 1961-1985. https://doi.org/10.1016/j.jbankfin.2003.07.006.

Taylor, M. (1988). An empirical examination of long-run purchasing power parity using cointegration techniques. Applied Economics, 20(10), 1369-1381. https://doi.org/10.1080/00036848800000107.

Urrutia, M., \& Llano, J. (2012). Los actores en la crisis económica de fin de siglo. Bogotá: Ediciones Uniandes.

Varian, H. (1992). Microeconomic analysis. New York: W.W. Norton \& Company, Inc.

Wang, P. (2009). The Economics of Foreign Exchange and Global Finance. Berlín, Alemania: Springer. 